\title{
Uso de la Kaizen para la mejora continua de la docencia de la asignatura Expresión Gráfica II Use of the Kaizen fot the continuous improvement of the teaching of the subject Graphical Expression II
}

\author{
Ramon Miralbes Buil, Pedro Valentín Ubieto Artur, Juan Antonio Peña Baquedano \\ mirales@unizar.es, pubieto@unizar.es, juanp@unizar.es \\ Ingenieria de Diseño y Fabricación \\ Universidad de Zaragoza \\ Zaragoza, España
}

\begin{abstract}
Resumen- El objetivo de este artículo es reflejar la metodología utilizada para la mejora de una asignatura del Espacio Europeo de Educación Superior (EEES) utilizando diversas herramientas de mejora continua, basado en el Kaizen. La mejora continua es una herramienta de trabajo especialmente conocida dentro del ámbito de la ingeniería, principalmente para la mejora de procesos y productos, pero que puede utilizarse en cualquier proceso de mejora. Concretamente, se han utilizado dos herramientas de esta metodología: el DAFO para el análisis de debilidades, amenazas, fortalezas y oportunidades y, a partir de los resultados obtenidos, se plantean diversas acciones con el objetivo corregir las debilidades, afrontar las amenazas, mantener las fortalezas y explotar las oportunidades, mediante lo que se conoce como herramienta CAME. Todo ello se ha implementado para la mejora del proceso de enseñanza-aprendizaje en la asignatura Expresión Gráfica II (EGII) del Grado de Ingeniería de Diseño Industrial y Desarrollo de Producto (IDIDP) de la Universidad de Zaragoza (UZ)
\end{abstract}

\section{Palabras clave: mejora continua, ingeniería, DAFO, CAME}

Abstract- The aim of this paper is to show the use of a methodology to improve a subject of the Superior European Education Space based on the use of diverse tools of the continuous improvement system, based on the Kaizen. The continuous improvement system is a tool specially used in the engineering sector to improve industrial process and products, but that can be used in any improvement process. Concretely, it has been used two tools: the SWOT and the CAME analysis that are complementary. The SWOT tool allows to determine the strengths and the weakness and to identify the opportunities and the threats. On the other hand, the CAME tool allows correcting the Weaknesses, adapting to and adjusting to the threats, maintaining the strengths and exploring the opportunities. These tools have been used to improve the teaching and the learning process in the subject Graphical Expression II of degree of Industrial Design and Product Development Engineering of the University of Zaragoza.

\section{Keywords: continious improvement, engineering, SWOT, CAME}

\section{INTRODUCCIÓN}

La docencia de cualquier asignatura es un proceso vivo que tiene y debe renovarse continuamente para adaptarse a las necesidades del alumnado y de la sociedad; de la misma forma el desarrollo tecnológico influye en el estado del conocimiento $\mathrm{y}$ en las necesidades de la sociedad $\mathrm{y}$, por tanto, en los conocimientos que deben tener los futuros profesionales para adaptarse a estos cambios de forma que los conocimientos y capacidades adquiridos en la Universidad se adapten a cada época y momento.

Es por ello que cualquier asignatura está habitualmente inmersa en un proceso continuo de mejora que se fundamenta en las percepciones del profesor, los comentarios de los alumnos, principalmente reflejados en las encuestas y verbalmente, y en el avance de los conocimientos y de la aplicación de los mismos a la sociedad, también en continuo cambio.

Uno de los aspectos más complicados para realizar este proceso continuo de mejora, es la correcta identificación de la situación interna de una asignatura, que permite recabar la información necesaria para poder implementar mejoras en función de un análisis concienzudo de esta información. Otro de los aspectos fundamentales, es la obtención de conclusiones en función de la información obtenida y la implementación de acciones, tanto correctivas y paliativas, para aquellos aspectos negativos, como acciones de refuerzo y potenciación en los aspectos positivos.

Para ello, tal como indican algunos autores como Trujillo, las herramientas del Kaizen y, en especial, los análisis DAFO y CAME, resultan de una gran utilidad y aplicabilidad, por lo que han sido aplicadas a un caso concreto de la asignatura Expresión Gráfica II

\section{CONTEXTO}

El artículo se centra en la asignatura Expresión Gráfica II (EG II) que desarrolla principalmente contenidos de Dibujo Industrial en el ámbito de la ingeniería, en concreto en el Grado de Ingeniería de Diseño Industrial y Desarrollo de Producto (IDIDP) de la Universidad de Zaragoza, con una carga lectiva de 6 créditos ECTS. Cabe señalar, que existen asignaturas similares en otros grados de la misma Universidad, en otras titulaciones como en el Grado de Ingeniería Mecánica (6 créditos) y en el Grado en Ingeniería Industrial (3 créditos), por lo que, los resultados y conclusiones obtenidos de este trabajo, pueden extrapolarse a otras titulaciones. Para realizar 
el estudio participaron los alumnos de la titulación IDIDP que suman un total de 84 alumnos para el curso 2016/2017. Los resultados se compararon con los resultados de cursos anteriores del mismo grado y con la misma cantidad de alumnos, y con otros grados, concretamente el Grado de Ingeniería Mecánica (IM) (4 clases de 60-70 alumnos) y el Grado de Tecnologías Industriales (ITI) (3 clases de 70-80 alumnos) en los que se realizó una docencia tradicional y con la que se compararon los resultados obtenidos.

La asignatura en los diversos grados mencionados, tiene una carga lectiva de 60 horas y se vertebra en forma de actividades de tipo teórico o clases magistrales (30 h), clases de problemas (15 h) y prácticas de laboratorio (15 h). Este esquema y distribución se debe fundamentalmente al esquema del calendario del cuatrimestre compuesto por quince semanas lectivas.

Debe señalarse que en todas las titulaciones indicadas, la asignatura correspondiente, se sitúa en el segundo curso del grado precedida de la asignatura previa Expresión Gráfica y Diseño Asistido por Ordenador, desarrollada en el primer curso. En esta asignatura, se desarrollan diversos y contenidos necesarios para la asignatura objeto del estudio, principalmente aspectos de normalización y croquizado. También existe una clara relación de la asignatura con la asignatura Expresión Artística, también de primer curso, donde se desarrollan diversos contenidos de croquizado y representación espacial. Es por ello, que debe tenerse en consideración dichas asignaturas, para un estudio de los conocimientos previos de los alumnos.

Otro aspecto a considerar, es los conocimientos que otras asignaturas del grado necesitan previamente, relacionados con la asignatura EG II para desarrollar sus contenidos. En este aspecto, la principal asignatura relacionada es la asignatura Oficina Técnica de cuarto curso.

En lo referente a las asignaturas que forman parte del mismo curso y cuatrimestre que EG II, en este caso primer cuatrimestre del segundo curso, debe señalarse que entre las mismas se realiza un trabajo conjunto y multidisciplinar, conocido como "trabajo de módulo", que permite a los alumnos conocer y aplicar los conocimientos y capacidades aprendidas en las diversas asignaturas a un trabajo conjunto y grupal. Con ello, se persigue la integración de conocimientos y el desarrollo de un proyecto desde un punto de vista multidisciplinar que integre a las diversas asignaturas.

Este proyecto de módulo está orientado fundamentalmente a la solución práctica de un caso concreto relacionado con el ámbito profesional como puede ser el re-diseño de un vehículo de tracción humana, el diseño de una trona, etc. por lo que es uno de los elementos vertebradores de la titulación y resulta de especial importancia, tal como indica Miralbes et. Al (2011).

Este trabajo permite aplicar los conocimientos adquiridos de forma teórica en cada asignatura desde un punto de vista multidisciplinar, conociendo la relación entre los diversos campos de conocimiento y su influencia tanto directa como cruzada, todo ello aplicado a un trabajo similar al desarrollado durante el futuro ejercicio profesional de la titulación.

Adicionalmente, cabe señalar que el trabajo permite desarrollar diversas capacidades como el trabajo en grupo, el liderazgo, la capacidad de análisis y de síntesis y mejora aspectos como la capacidad de hablar en público.
Tras el sexto año de implantación del segundo curso del grado aparece, debido al proceso de acreditación de las titulaciones en la ANECA, la necesidad de plantear modificaciones en el grado a partir de la experiencia en los últimos seis años. Es por ello que se produce una reestructuración del grado de forma que, concretamente en el segundo curso primer cuatrimestre, se producen cambios significativos relacionados con las asignaturas con las que comparte docencia EGII y que aparecen reflejadas en la tabla I; adicionalmente, la tabla muestra la contribución de cada asignatura relacionada con el trabajo de módulo.

\begin{tabular}{|l|l|}
\hline Asignaturas 2015/2016 & Asignaturas 2016/2017 \\
\hline $\begin{array}{l}\text { Diseño Asistido por } \\
\text { Ordenador I (9 cred): } \\
\text { modelado 3D }\end{array}$ & $\begin{array}{l}\text { Diseño Asistido por } \\
\text { Ordenador I (6 cred): ídem }\end{array}$ \\
\hline $\begin{array}{l}\text { Mecánica (9 cred): diseño } \\
\text { estructural }\end{array}$ & $\begin{array}{l}\text { Diseño de Mecanismos (6 } \\
\text { cred): diseño cinemático y } \\
\text { dinámico }\end{array}$ \\
\hline $\begin{array}{l}\text { Taller de Diseño II (6 cred): } \\
\text { diseño conceptual y } \\
\text { desarrollo del producto }\end{array}$ & $\begin{array}{l}\text { Taller de Diseño II (6 cred): } \\
\text { ídem }\end{array}$ \\
\hline $\begin{array}{l}\text { Expresión Gráfica II (6 } \\
\text { cred): representación 2D del } \\
\text { producto de acuerdo a la } \\
\text { normalización }\end{array}$ & $\begin{array}{l}\text { Expresión Gráfica II (6 } \\
\text { cred): ídem }\end{array}$ \\
\hline & $\begin{array}{l}\text { Expresión Artística II (6 } \\
\text { cred): representación } \\
\text { mediante maquetas virtuales } \\
\text { y reales, así como mediante } \\
\text { paneles de presentación }\end{array}$ \\
\hline
\end{tabular}

\section{Tabla I: Asignaturas de $2^{\circ}$ Curso 1er cuatrimestre Grado} IDIDP

Otro aspecto fundamental a tener en cuenta para la implementación de cualquier mejora, es el estudio del alumnado de la titulación que debe tenerse en consideración. En el caso particular abordado, existen estudios previos como el de Serrano Et. Al (2016) que analiza en profundidad este aspecto y que ha sido contemplado para el estudio.

\section{DESCRIPCIÓN}

El Kaizen, conocido habitualmente en español como mejora continua, es un método de gestión de calidad de proveniencia japonesa, muy conocido en el mundo industrial y que se basa en los principios del taoísmo y del budismo.

El objetivo del Kaizen es, tal como indica el fundador del Instituto del Kaizen, Masaaki Imai (2014) aportar productos de una mayor calidad, variedad y menor coste y tiempo de respuesta; todo ello dentro de un mundo en constante y cada vez más acelerados cambios, que exigen la reducción del ciclo de vida de los productos, la competencia global y la evolución constante de los hábitos de los consumidores. Para ello se debe involucrar a todos los niveles de la empresa, desde la gerencia hasta el personal de limpieza

En esta idea de la mejora continua, se involucra tanto la gestión como el desarrollo de procesos, enfatizando las necesidades de los clientes con el objetivo de reconocer y reducir los desperdicios a la vez que se maximiza el tiempo. 
Es por ello que, como en la filosofía “Just in Time”, el tiempo adquiere una importancia primordial.

Esta filosofía es extrapolable al ámbito de la educación superior, donde el producto es los conocimientos y capacidades que debe adquirir el alumnado y que, totalmente condicionado por el continuo cambio tecnológico y social de una sociedad tecnológica y post industrial como la actual.

Con la irrupción de los rankings de universidades y la globalización, la educación superior empieza a sufrir un proceso de competencia global que generará una alta competencia entre universidades, tal como ocurre en el mundo anglosajón. Adicionalmente, cada vez existe más información relacionada con la docencia universitaria por lo que los estudiantes tienen una mayor capacidad para seleccionar la universidad de destino, lo que implica una mayor competencia entre universidades que se agravará con los efectos demográficos de una sociedad post industrial.

Desde el punto de vista del tiempo, con el aumento de las necesidades de aprendizaje y contenidos, resulta imprescindible la optimización de tiempos dentro del ámbito de la educación superior, para poder alcanzar unos objetivos concretos y satisfacer unas necesidades de conocimiento cada vez mayores. Es por todo ello que la filosofía del Kaizen puede mejorar de forma importante la docencia de cualquier asignatura $\mathrm{y}$, en un rango más amplio, la docencia de una determinada titulación y universidad.

Dos de las herramientas principales del Kaizen son el análisis DAFO, que permite analizar la situación actual de cualquier proceso, y el análisis CAME, que permite posteriormente realizar una serie de acciones encaminadas a la mejora continua de cualquier proceso.

El análisis DAFO, tal como indica Martínez, D. (2012) realiza un análisis tanto interno como externo de un determinado proceso o situación. El análisis externo centro su atención en el estudio del entorno que condiciona inherentemente el proceso. En este aspecto, repercuten aspectos de carácter tecnológico, legal, político y social que pueden influir en el proceso o en la organización tanto positiva como negativamente por lo que aparecen las Oportunidades que se deben aprovechar y las Amenazas para las que deben planificarse estrategias para minimizarlas y/o evitarlas.

Desde un punto de vista interno, el estudio permite conocer los recursos y procesos, tanto en calidad como en cantidad con los que cuenta; con ello, se pueden identificar atributos que generan una ventaja o una desventaja con respecto a los competidores que, en este caso, serían otras asignaturas de otras universidades o de la misma universidad. Cabe señalar, que la competitividad entre asignaturas y universidades genera finalmente un proceso de mejora continua. Finalmente, este estudio permite fijar las fortalezas para poder explotarlas y las debilidades para poder subsanarlas.

Una vez realizado el análisis DAFO, la siguiente fase es el desarrollo de un análisis CAME, que estudia cómo Corregir las Debilidades, Afrontar las Amenazas, Mantener las Fortalezas y Explotar las Oportunidades.

Debe señalarse que, habitualmente, tanto el análisis DAFO como el CAME se presentan mediante sendos cuadro de 2x2 tal como indica a modo ilustrativo la tabla II, pero que, debido al formato del artículo, se van a presentar en forma de columnas verticales.

\begin{tabular}{|c|c|c|}
\hline $\begin{array}{l}\text { Análisis } \\
\text { Interno }\end{array}$ & $\begin{array}{c}\text { Fortalezas } \\
\text { distintas. } \\
\text { Ventajas naturales } \\
\text { Recursos superiores }\end{array}$ & $\begin{array}{c}\text { Debilidades } \\
\text { Recursos y capacidades } \\
\text { escasas }\end{array}$ \\
$\begin{array}{c}\text { Oportunidades } \\
\text { Problemas de motivación }\end{array}$ \\
\hline $\begin{array}{c}\text { Análisis } \\
\text { Externo }\end{array}$ & $\begin{array}{c}\text { Nuevas tecnologías } \\
\text { Debilitamiento de }\end{array}$ & $\begin{array}{c}\text { Altos riesgos - Cambios } \\
\text { en el entorno }\end{array}$ \\
\hline \multicolumn{2}{|c|}{ Tabla II: Ejemplo de Análisis DAFO. Fuente: Wikipedia. }
\end{tabular}

Tal como se ha indicado anteriormente, la metodología de docencia propuesta se ha aplicado a toda una clase de un determinado grado, en este caso el grado de IDIDP con el objetivo de no utilizar metodologías ni criterios diferentes dentro de una misma titulación ni explicar contenidos diferentes y se ha aplicado durante el curso 2016/2017 pero se prevé su aplicación en el futuro en otros grados y la continuación de la utilización de la misma metodología para el próximo curso. Es por ello que la muestra de estudiantes utilizado ha sido de 84 .

Para realizar el estudio comparativo de resultados, se han utilizado de grupo de control los estudiantes del resto de titulaciones de la misma universidad, en concreto los de los grados de IM y de ITI, con 4 y 3 grupos respectivamente de 60-80 alumnos. En estas clases, se ha realizado una docencia tradicional pero se ha utilizado la misma prueba final para analizar comparativamente los resultados de aprendizaje adquiridos.

Adicionalmente se han utilizado los datos históricos de la asignatura de los cursos 2008/2009 a 2015/2016. En concreto se han utilizado las encuestas de evaluación, los resultados de las pruebas de evaluación y de la evaluación de los trabajos y la nota final.

En relación a las encuestas utilizadas como método de evaluación de la metodología propuesta, no se han desarrollado encuestas específicas sino que se ha recurrido a las encuestas de evaluación, tanto de la labor docente como de la propia asignatura, de la Universidad de Zaragoza (ver página web). Estas encuestas son de cumplimentado optativo por parte del alumnado y se han mantenido inalteradas durante los últimos 6 cursos (participación media 30-40\%) y permiten realizar estudios comparativos entre titulaciones, entre asignaturas de la misma titulación y entre cursos diferentes,

\section{Resultados}

A continuación se van a mostrar en forma de una única columna el análisis DAFO en la tabla III. Los datos se han obtenido a partir de las encuestas y entrevistas con los alumnos, de la memoria de verificación de la ANECA correspondiente al cambio de la titulación y de los comentarios de otros alumnos y profesores de las diversas titulaciones en las que se imparte esta docencia (ITI, IDIDP e IM). 


\section{Debilidades}

Poco interés por parte del alumno (Teoría).

Percepción del alumno de escasa conexión con el mundo laboral.

Contenidos desactualizados.

Falta de algunos contenidos importantes y de profundización en otros.

Trabajo de módulo ambicioso y extenso

Prácticas desactualizadas (AutoCAD) y poco útiles según los alumnos

\section{Amenazas}

Trabajo de asignatura igual en todos los grupos $\rightarrow$ control del plagio de trabajos.

Trabajo de módulo ambicioso carga muy elevada de trabajo el final del cuatrimestre $\rightarrow$ poca dedicación a la asignatura.

Distribución del trabajo de módulo a criterio del alumnado $\rightarrow$ se concentra el trabajo al final.

EGII planos mediante herramientas 2D / DAO de planos con herramientas $3 \mathrm{D} \rightarrow$ poco control.

\section{Fortalezas}

Trabajo de módulo multidisciplinar $\rightarrow$ percibido como útil e interesante por los alumnos. Identificado de forma positiva por la ANECA.

Trabajo de asignatura percibido como útil y con gran aplicabilidad práctica.

Uso de rúbricas en módulo $\rightarrow$ evaluación clara y automatizada y percibida como clara y “justa”.

Evaluación por ítems del examen $\rightarrow$ baja cantidad de reclamaciones/percepción buena.

Trabajo de asignatura mediante 2 entregas $\rightarrow$ trabajo continuo del alumnado

\section{Oportunidades}

Uso de herramientas web: Moodle, videos de You Tube, plataforma propia para rúbricas, etc.

Trabajo de módulo con nuevas asignaturas más cercanas (Diseño de Mecanismos).

Cambio en la carga de docencia de la asignatura Diseño Asistido por Ordenador I ( 9 a 6 créditos) $\rightarrow$ reducción de los contenidos de DAO.

Coordinación de ña docencia con DAO y Diseño de Mecanismos $\rightarrow$ contenidos similares.

\section{Tabla III: Análisis DAFO de la asignatura.}

A partir del análisis DAFO, se han planificado, siguiendo con la filosofía del Kaizen, diversas acciones, plasmadas en el análisis CAME que se muestra en la tabla IV. Para realizar ambas tabla se han realizado diversas sesiones de Brain Storming involucrando a los profesores de la asignatura en las diversas titulaciones, a alumnos de los dos cursos anteriores y a profesores de asignaturas relacionadas y del mismo cuatrimestre y se han obtenido datos y propuestas de las encuestas de evaluación de los cursos anteriores de la misma y de otras titulaciones.

\section{Corregir las Debilidades}

Nuevas metodologías: portafolio y learning by doing.

Aproximación al mismo: trabajo de asignatura y de módulo.

Actualización de contenidos: revisión de normas.

Aumento de contenidos y profundización.

Simplificación de trabajo de módulo: diseño de una atracción de feria $\rightarrow$ re diseño de un Kart.

Prácticas en Inventor: planos a partir de modelado 3D.

\section{Afrontar las Amenazas}

Trabajos de asignatura diferentes en cada grupo.

Simplificación del trabajo de módulo. Mejor distribución en tiempo del mismo. Obligatoriedad de adelantar la parte “Creativa” en Taller de Diseño.

Reorganización y planificación del trabajo de módulo $\rightarrow$ presentación intermedia de la parte de diseño y concepción de mecanismos.

Desarrollo de la parte de planos a partir del 3D en la asignatura $\rightarrow$ coordinación con DAO I

\section{Mantener las Fortalezas}

Mantener trabajo de módulo pero simplificado.

Aumentar trabajo de asignatura $\rightarrow$ modelado + planos

Rúbricas en evaluación de trabajo y prácticas.

Mantener la evaluación por ítems del examen.

Mantener 2 entregas del Trabajo de asignatura.

\section{Explotar las Oportunidades}

Uso de Moodle (encuestas, selección trabajos, apuntarse prácticas) YOU TUBE (selección de videos relacionados), rubrica accesible y visible a través de web.

Mejorar de la coordinación de Trabajo de módulo.

Prácticas: planos en 3D a partir de modelado.

Coordinación de prácticas con DAO y contenidos con Diseño de Mecanismos.

\section{Tabla IV: Análisis CAME de la Asignatura}

Tras la implantación de las diversas mejoras indicadas durante el curso 2016/2017 y, tras las pruebas de evaluación de febrero, se ha constatado una mejora en los resultados de la asignatura, de forma que el porcentaje de aprobados en primera convocatoria (media de los últimos 6 cursos) ha pasado del 53\% al 64\%. Adicionalmente, se ha constatado un aumento los conocimientos adquiridos por el alumnado, que han quedado plasmados en el trabajo de asignatura.

Para realizar dicha evaluación se han comparado de forma ciega con un grupo de profesores ajeno a la asignatura y al proyecto de innovación, pero de la misma área de conocimiento, trabajos de las diversas titulaciones anteriormente mencionadas. 
Actualmente, se dispone de los resultados de las encuestas que, comparado con las encuestas de años posteriores, refleja una mejora, tanto en la percepción del alumno de la asignatura como de la labor docente del profesorado, que aparece reflejada en la tabla V.

\begin{tabular}{|l|l|l|l|l|}
\hline $\begin{array}{l}\text { Resultados Encuesta } \\
\text { (1 mínimo-5 máximo) }\end{array}$ & 2013 & 2014 & 2015 & $\mathbf{2 0 1 6}$ \\
\hline Labor Docente & 3,45 & 3,63 & 3,42 & $\mathbf{4 , 2 8}$ \\
\hline Asignatura & 3,41 & 3,52 & 3,51 & $\mathbf{3 , 8 6}$ \\
\hline
\end{tabular}

Tabla V: Evolución de la valoración de la asignatura y de la labor docente

Tras el primer año de docencia, se han obtenido las siguientes conclusiones positivas: el plagio de trabajos se ha reducido drásticamente (2015/2016: 4 trabajos/40 $\rightarrow$ 2016/2017: 1 trabajo/25), la profundidad, calidad y complejidad de los trabajos ha aumentado considerablemente, se ha constatado una actitud más activa por parte del alumnado en las clases teóricas y una mayor comprensión de la asignatura y de su aplicación práctica. Además, con la simplificación del trabajo de módulo y su correcta aplicación y distribución espacial, ha sido posible realizar un trabajo de asignatura específico de mayor complejidad; también se ha constatado una mayor utilidad de las prácticas de laboratorio y se han relacionado contenidos y coordinado esfuerzos con otras asignaturas lo que ha redundado positivamente en todas ellas. Adicionalmente, se ha reducido el absentismo tanto por el carácter práctico como por el hecho de proporcionar enunciados de problemas sin resolución a través de Moodle (anteriormente se incluía la resolución por lo que un porcentaje del alumno no creía necesario asistir a clase).

Como puntos a mejorar, se ha constatado que el alumnado de forma generalizada no realiza los ejercicios propuestos ni estudia previamente la teoría sugerida, por lo que se plantea la obligatoriedad de la entrega de los mismos. También se ha constatado que la asistencia durante una práctica al laboratorio de "elementos y conjuntos" ha sido positiva y muy bien valorada por el alumnado por lo que se plantean para el próximo curso seminarios quincenales de 30 minutos para utilizar dicho laboratorio donde se puede "tocar” y desmontar elementos reales.

\section{CONCLUSIONES}

El trabajo propuesto demuestra cómo diversas herramientas del ámbito industrial integradas en el Kaizen, pueden aplicarse para mejorar cualquier ámbito de la docencia universitaria, tanto una asignatura concreta como una titulación o la gestión de un espacio o de un laboratorio.

En este caso concreto, se han utilizado para la mejora de la docencia de una determinada asignatura de carácter técnico, pero, tal como establece la filosofía del Kaizen, la forma de implementación óptima sería en todos los ámbitos de una institución universitaria o en su defecto en toda una titulación.

Con ello, se podría realizar una mejor implementación pero, para ello, resulta fundamental la implicación activa de todos los miembros de la comunidad universitaria, lo que resulta de especial dificultad. Se debe señalar que éste es uno de los aspectos que presenta una mayor complejidad y, aunque en este caso concreto sólo implicaba a una asignatura con una única clase, no ha sido posible implicar a todos los profesores de la misma.

En relación a las recomendaciones para la aplicación del proceso de mejora continua, resaltar que es un proceso vivo y que debe intentar realizarse de forma progresiva. En el caso planteado. los objetivos planteados inicialmente han sido muy ambiciosos lo que ha repercutido en una gran carga de trabajo para el profesorado. Es por ello que se recomienda la implementación progresiva con objetivos iniciales modestos, que puedan ampliarse de forma progresiva para evitar el desánimo y la saturación del profesorado.

Finalmente concretar que en lo referente a la asignatura en la que se ha implementado la mejora, se ha conseguido aumentar la asistencia a clase, reducir el plagio, mejorar la percepción del estudiante de la asignatura, su aplicabilidad y su utilidad, optimizar la carga lectiva, aumentar los contenidos de la asignatura y los conocimientos adquiridos.

\section{REFERENCIAS}

Imai, M. (2014). Gemba Kaizen: un enfoque de Sentido común para una Estrategia. McGraw-Hill.

Martínez, D. (2012). Diagnóstico Estratégico. Harlequin Ibérica.

Miralbes, R., Auria, J.M., Tardío, E. y López, I. (2011). Experiencia innovadora en la docencia de las asignaturas del grado de ingeniería de diseño industrial y desarrollo de producto basada en actividades multidisciplinares. Arbor, 187/3.

Serrano, A., Biedermann, A. y Santolaya, J.L. (2016). Objetivos, competencias y expectativas de futuro profesional de los estudiantes del Grado en Ingeniería en Diseño Industrial y Desarrollo de Producto. REDU. Revista de Docencia Universitaria, 14/1.

Trujillo, F. "El análisis DAFO en el diseño de proyectos educativos: una herramienta empresarial al servicio de la educación”. http://www.educacontic.es/blog/el-analisisdafo-en-el-diseno-de-proyectos-educativos-unaherramienta-empresarial-al-servicio

https://es.wikipedia.org/wiki/An\%C3\%A1lisis_DAFO

https://encuestas.unizar.es/ 